\title{
FORENSIC PSYCHIATRIC ASSESSMENT OF THE CRIMINAL AND CIVIL RESPONSIBILITY OF MENTALLY DISORDERED PERSONS
}

\author{
Safaa. Y. Eldabe 1, Amal. A. Ahmed 2, Ayat. A. Hemeda3, Adel. A. El \\ Zaid4 and Ahmed. M. Hamoda5
}

\author{
1 Professor, 2 Assistant Professor, 3Assistant Lecturer \\ 1, 2, 3 Forensic Medicine and Clinical Toxicology Department, \\ Faculty of Medicine for Girls, Al-Azhar University, Cairo, Egypt \\ 4Consultant Psychiatrist, Medical and General Director of Kuwait Center for Mental \\ Health 5Neuropsychiatry specialist and previous Assistant Director of Abbasia \\ Hospital for Mental Health \\ 4, 5Kuwait Centre for Mental Health
}

\begin{abstract}
Background: The psychiatric assessment of individuals involved in legal proceedings had significantly been advanced during the past decades. Aim of study: To estimate the prevalence of criminal and civil problems among mentally disordered persons, identify their characteristics and determine factors that may be predictive of their responsibilities. Participants and Methods: This study was a prospective one that included 600 cases who were referred from different departments of the legal system to the forensic psychiatry unit for assessment of their criminal or civil responsibilities over 15-months duration. Data related to the cases as regarding their characteristics, diagnosis, history of illness and history of substance abuse were collected. Cases were classified into criminal and civil groups and comparison between the two groups was performed. Statistical analysis: Analysis of collected data were done by using SPSS program version 19. Results: Most of cases (86\%) presented with civil problems, while $14 \%$ of the cases presented with criminal problems. Males represented $91.7 \%$ of criminal and $61.6 \%$ of civil cases. The mean age was 33.38 years in criminal and 36.83 years in civil cases. Most of criminal and civil cases were unemployed (86.7\%) and single (64.3\%). High percentage of criminal cases $(45.2 \%)$ were substance abusers, had history of crimes $(53.6 \%)$. $42.9 \%$ of criminal cases had no social support. $48.8 \%$ of criminal cases were considered responsible for their crimes and $85.6 \%$ of civil cases were considered non-competent. There were statistically significant differences in the criminal and civil responsibilities as regarding diagnosis, severity of mental illness

and

degree of cognitive impairment. Conclusions: Comorbidity with substance use disorder and absence of social support are risk factors of criminal behaviour in mentally disordered persons. The criminal and civil responsibilities in mentally disordered persons are affected by the diagnosis,
\end{abstract}


severity of mental illness and the degree of cognitive impairment. Recommendations: Cooperation should be established among the criminal justice, social services and medical systems to reduce criminal behaviour among persons with mental disorders through early detection, treatment, rehabilitation and reducing the incidence of substance abuse among them.

Key Words: Forensic Psychiatry, Mental illness, Criminal, Civil, Responsibility,

Substance abuse.

\section{INTRODUCTION}

Mental disorder is an illness with psychological or behavioral manifestations associated with significant distress and impaired functioning caused by a biological, social, psychological, genetic, physical, or chemical disturbance. It is measured in terms of deviation from some normative concept (Sadock and Sadock, 2005).

Mental disorders are highly prevalent in all regions of the world and represent a major source of disability and social burden worldwide. The World Health Organization (WHO) estimates that more than 25 percent of individuals worldwide develop one or more mental disorders during their lifetime (Sadock et al., 2015).

A National Survey of Prevalence of Mental Disorders in Egypt found that, mental disorders were estimated at $16.93 \%$ of the studied adult population (Ghanem et al., 2009).

Mental illnesses can cause social isolation, poor quality of life, increased mortality, and are related to many other health concerns. Mental illnesses also carry tremendous economic and social costs (WHO, 2007).
"Forensic Psychiatry is a subspecialty of psychiatry in which scientific and clinical expertise is applied in legal contexts involving civil, criminal, correctional, regulatory or legislative matters, and in specialized clinical consultations in areas such as risk assessment or employment" (AAPL, 2005).

Forensic psychiatry should be practiced in accordance with guidelines and ethical principles enunciated by the profession of Psychiatry (Nambi et al., 2016).

Various reasons for forensic psychiatric evaluation can be broadly grouped under criminal and civil groups. A criminal court may ask for assessing the fitness of a person to stand trial or determining the criminal responsibility of a person who committed a crime while, the civil court may require psychiatric assessment to determine the mental capacity required for competency in many civil issues including cases of guardianship, to know whether a person with mental illness is able to take care of self and manage his/her affairs or needs a guardian, also in issues of testamentary capacity, marital 
dispute and divorce on grounds of mental illness , child custody, disability compensation, fitness for work, fitness to give a consent or to enter in a contract (Chadda et al., 2002).

abuse, or a criminal record in the family in addition to, mental status examination and personality assessment. Minor modifications in the assessment format may be required depending on the kind of request (Klassen \& Wright, 2006).

Arab countries were among the first in the world to establish mental health hospitals (in Baghdad in the year 705, Cairo in 800 and in Damascus in 1270) (Youssef et al., 1996), but currently most Arab countries have no mental health acts, no certified training in forensic psychiatry, there is little research in forensic psychiatry and forensic psychiatric services are poorly organized (Okasha, 2003).

\section{PATIENTS AND METHODS}

This study was performed at "Kuwait centre for Mental Health" which is managed by the Ministry of Health of Kuwait. The centre is recognized for teaching the Kuwaiti board of psychiatry in addition, it is the service and teaching arm of the Department of Psychiatry at Faculty of Medicine, Kuwait University. The forensic psychiatry unit is one of the most important units inside the centre All cases required forensic psychiatric assessment in Kuwait
Forensic psychiatric assessments should include taking information regarding socioeconomic status of the individual, personal history of psychiatric illness, forensic history,

state are directed to forensic psychiatry unit at "Kuwait centre for Mental Health". The forensic psychiatry committee consists of psychiatrists, psychologists, social workers and nurses. Most of members of the team were experienced in forensic psychiatry for many years.

This study was approved by the scientific and ethical committee of the Ministry of Health of Kuwait.

\section{- Selection of the sample}

This is a prospective study conducted on 600 cases of forensic psychiatry which were referred from different departments of the legal system (police, prisons, courts, general department of crime evidence and state security) to the forensic psychiatry unit at "Kuwait centre for Mental Health" for assessing possible mental disorders at the period from first April 2016 to the end of July 2017.

\section{- Inclusion criteria:}

- Adult males and females patients $>18$ years having mental or personality disorders.

- Patients referred for assessment of their criminal responsibility. 
- Patients referred for assessment of their civil competency

- Exclusion criteria:

- Patients below 18 years.

- Malingering persons (excluded by examination of forensic psychiatric committee).

- Data collection:

- Data related to the cases as regarding their sociodemographic characteristics, diagnosis, Substance abuse, Crime details, present mental illness, past history of mental illness, past criminal history, family history, and the final opinion of the committee. These points were mentioned in Practice Guideline for the Forensic Assessment prepared by the American Academy of Psychiatry and the Law (AAPL, 2015). Diagnoses of mental disorders

Diagnoses of mental disorders were made according to the Diagnostic and Statistical Manual of Mental Disorders, fifth edition (DSM-5) diagnostic criteria American Psychiatric Association (APA, 2013).

Cases accused of committing crimes were classified according to type of crime into homicide, aggravated assaults, rape and sexual assault, kidnapping, threat, attempt of suicide, offences against properties, robbery, financial crimes, simple assaults (including quarrel and verbal assault), political crimes and, other crimes (drunk, possession of a weapon or sharp instrument, illegal country crossing, reckless car driving, enable an accused to escape from a lawful custody and, possession of illegal substances for purpose of abuse).

\section{- Statistical analysis:}

Statistical analysis of collected data were done by using SPSS program (statistical package of social science; SPSS Inc., Chicago, IL, USA) version 19 for Microsoft Windows. Mean and standard deviation were calculated to measure central tendency and dispersion of quantitative data. Comparison between two independent mean groups was done using Student $t$ test. Frequency of occurrence was calculated to measure qualitative data. Chi-square-test (2) was used for comparison of qualitative data and Fisher exact test was used instead for comparison of qualitative data of less than 5 in frequency. The level of significance was taken at $\mathrm{p}$-value of <0.05. The results were represented in tables.

\section{RESULTS}

The examined cases included 600 forensic psychiatry cases that were classified into $84(14 \%)$ criminal and $516(86 \%)$ civil cases Table (1).

\section{Criminal cases:}

The examined criminal cases were 84 offenders accused of committing various kinds of crimes and were referred to be 
assessed by forensic psychiatric committee to determine if they had any mental disorder and to assess their criminal responsibility. 5 offenders $\quad(5.9 \%) \quad$ committed homicide, 7 offenders (8.3\%) committed aggravated assaults, 6 offenders $(7.1 \%)$ committed rape and sexual assault, 2 offenders (2.4\%) committed kidnapping, 4 offenders $(4.8 \%)$ committed threat (menace), 4 offenders $(4.8 \%)$ committed attempt of suicide, 17 offenders $(20.2 \%) \quad$ committed offences against properties, 4 offenders $(4.8 \%)$ committed robbery, 4 offenders (4.8\%) committed financial crimes, 17 offenders $\quad(20.2 \%) \quad$ committed Simple assaults (including quarrel and verbal assault), 7 offenders (8.3\%) committed Political crimes and 7 offenders $(8.3 \%)$ committed other crimes (drunk, possession of a weapon or sharp instrument, illegal country crossing, reckless car driving, enable an accused to escape from a lawful custody, possession of illegal substances for purpose of abuse).

\section{Civil cases:}

The number of the examined civil cases was 516, these cases were referred to determine if they had any mental disorder and to assess if the mental disorder affect their mental capacity required for competency in a particular task. 480 cases $(93 \%)$ were assessed for the purpose of guardianship, 26 cases $(5 \%)$ were assessed for both guardianship and determination of disability degree, 4 cases $(0.8 \%)$ were assessed for both disability degree and their fitness for work, 3 cases $(0.6 \%)$ were assessed for child custody and 3 cases $(0.6 \%)$ were assessed for purpose of nullity of a contract.

- Demographic characteristics of the examined criminal and civil cases:

In this study $91.7 \%$ of criminal cases and $61.6 \%$ of civil cases were males (Chi-square test $=$ 28.98, $\mathrm{P}$ value $=0.000$ ). The mean age was $33.38 \pm 9.57$ years in criminal cases and $36.83 \pm 18.12$ years in civil cases (student $\mathrm{t}$ test= $1.70, \mathrm{P}$ value $=0.089$ ). Cases had educational level lower than secondary school represented $36.9 \%$ of criminal cases and $45.5 \%$ of civil cases, cases had secondary school or technical institutes educational level represented $44 \%$ of criminal cases and $32.4 \%$ of civil cases, university and post graduate educational level represented $19.1 \%$ of criminal and $22.1 \%$ of civil cases (Chi-square test $=4.43, \mathrm{P}$ value $=0.109)$. $67.9 \%$ of criminal cases and $89.7 \%$ of civil cases were unemployed (Chi-square test $=29.9, \mathrm{P}$ value $=$ 0.000). Half of criminal cases $(50 \%)$ and most of civil cases $(66.7 \%)$ was single (Fisher's exact $=23.6, \mathrm{P}$ value $=0.000)$. Table (2).

- Diagnosis of the examined criminal and civil cases:

There was a statistically significant difference between criminal and civil cases regarding all the diagnostic parameters with 
statistically significant higher percent of intellectual disability and neurocognitive disorder in civil cases and statistically significant higher percent of schizophrenia, bipolar I disorder and comorbidity with substance use disorder in criminal cases $(\mathrm{p}=$ $0.000 *$ for all) Table (3).

- Substance abuse of the examined criminal and civil cases:

There was a statistically significant difference between criminal and civil groups as regarding substance abuse with higher percentage of substance abusers in criminal cases $(45.2 \%)$ while, most of civil cases $(98.3 \%)$ were non-abusers $(\mathrm{p}=0.000 *)$ Table (4).

- Past history of mental illness of the examined criminal and civil cases:

The mean duration of illness was 12.67 years in criminal cases while 21.87 years in civil cases. Most criminal cases $(59.5 \%)$ had past history of admission in psychiatric hospital, while most civil cases $(76 \%)$ had no past history of admission in psychiatric hospital. Most criminal cases $(86.5 \%)$ were non-compliant on treatment, while $65.1 \%$ of civil cases were compliant on treatment with a statistical significant difference between both groups $(\mathrm{p}=$ 0.000*) Table (5).
- Past criminal history of the examined criminal and civil cases:

There was a statistically significant difference between criminal and civil cases as regarding past criminal history as most criminal cases $(53.6 \%)$ had past history of crimes, while most civil cases (93.2\%) had no past history of crimes $(p=0.000 *)$ Table (6).

- Family history of the examined criminal and civil cases:

Most criminal cases (53.6\%) had family history of mental illness, while most civil cases $(65.3 \%)$ had no family history of mental illness. Family history of substance abuse and family history of crimes were presented in criminal cases in higher percentage than in civil cases. High percentage of criminal cases $(42.9 \%)$ had no social support, while most of civil cases $(86.2 \%)$ had good social support with a statistical significant difference between both groups $\left(\mathrm{p}<0.05^{*}\right.$ for all) Table (7).

- Final opinion of the committee in the examined criminal cases:

i. Opinion of the forensic psychiatry committee about the criminal responsibility of the examined criminal cases:

Table (8) showed the opinion of the forensic psychiatry committee where 41 
cases $(48.8 \%)$ were considered responsible while, 43 cases $(51.2 \%)$ were considered nonresponsible.

Most cases of homicide, rape and sexual assaults, kidnapping and financial crimes $(80 \%, 100 \%, 100 \%$ and $75 \%$ respectively) were considered responsible, while most cases of aggravated assaults, threats, offences against properties, simple assaults, political crimes and other crimes $(85.7 \%, 75 \%$, $64.7 \%, 52.9 \%, 57.1 \%$ and $57.1 \%$ respectively) were considered non-responsible, half cases of attempts of suicide and robbery were considered responsible and the other half were considered nonresponsible.

\section{ii. The relation between} criminal responsibility and diagnosis of the offenders:

There was a statistically significant difference in the criminal responsibility as regarding diagnosis of the offenders, most cases of schizophrenia, bipolar I disorder, and delusional disorder were considered non-responsible, while most cases of schizoaffective disorder, intellectual disability, major depressive disorder, personality disorders, comorbidity with substance use disorder and comorbidity with personality disorder were considered responsible $\left(\mathrm{p}=0.000^{*}\right)$ Table $(9)$.

\section{iii. The relation between} criminal responsibility and the state of mental illness:

There was a statistically significant difference in the criminal responsibility according to the severity of mental illness, degree of cognitive impairment, impairment of testing reality, impairment of self-control and impairment of insight $(\mathrm{p}$ $=0.002 *, 0.05 *, \quad 0.000 *$, $0.000 *$ and $0.000 *$ respectively) Table (10).

- Opinion of the committee in the examined civil cases:

As regarding cases of guardianship, the majority of the cases $394(82.1 \%)$, were considered non-competent and had no ability to be competent in the future and 17 cases (3.5\%) were considered noncompetent but may be competent later on while, 68 cases $(14.2 \%)$ were considered competent and in one case $(0.2 \%)$, the committee recommended to be evaluated after 6 months. Moreover, in cases of child custody and nullity of contract all cases were considered competent Table (11).

In cases of guardianship and disability degree, 20 cases (76.9\%) were considered noncompetent and their disability degrees were considered > $50 \%$, while 6 cases $(23.1 \%)$ 
were considered competent, in 3 of them the disability degrees were considered 10- 20\% and

Regarding the examined cases for fitness for work and disability degree; 3 of them (75\%) were considered fit for work, while one case (25\%) was considered not fit for work and the disability degrees were $10-20 \%$ in all of them Table (13).

- The relation between civil responsibility (competency) and the diagnosis in guardianship cases:

Table (14) showed that most cases of intellectual disability, schizophrenia, schizoaffective disorder, neurocognitive disorders, coma and in 3 cases the disability degrees were $20-50 \%$ Table (12).

cases with dual diagnosis were considered non-responsible (non-competent), while most cases with major depressive disorder, personality disorders, insomnia disorder were considered responsible (competent). This relation was statistically non-significant.

Table (15) showed a statistically significant difference in the civil responsibility (competency) in cases of guardianship according to the severity of mental illness and degree of cognitive impairment. 
Table (1): Legal classification of the examined forensic psychiatric cases

\begin{tabular}{|l|l|l|l|l|l|}
\hline Criminal Cases (84) & No & $\%$ & Type of civil Competency & No. & $\%$ \\
\hline Type of crime & 5 & $5.9 \%$ & Guardianship & 480 & $93.0 \%$ \\
\hline Aggravated assault & 7 & $8.3 \%$ & Guardianship \& Disability degree & 26 & $5.0 \%$ \\
\hline Rape and sexual assault & 6 & $7.1 \%$ & $\begin{array}{l}\text { Disability degree \& Fitness for } \\
\text { work }\end{array}$ & 4 & $0.8 \%$ \\
\hline Kidnapping & 2 & $2.4 \%$ & Child custody & 3 & $0.6 \%$ \\
\hline Threat (menace) & 4 & $4.8 \%$ & Nullity of a contract & 3 & $0.6 \%$ \\
\hline Attempt of suicide & 4 & $4.8 \%$ & & & \\
\hline Offences against properties & 17 & $\begin{array}{l}20.2 \\
\%\end{array}$ & & & \\
\hline Robbery & 4 & $4.8 \%$ & & & \\
\hline Financial crime & 4 & $4.8 \%$ & & & \\
\hline Simple assault & 17 & $\begin{array}{l}20.2 \\
\%\end{array}$ & & & \\
\hline Political crimes & 7 & $8.3 \%$ & & $\mathbf{5 1 6}$ & $\mathbf{1 0 0 \%}$ \\
\hline Other crimes & 7 & $8.3 \%$ & & & \\
\hline Total & $\mathbf{8 4}$ & $\begin{array}{l}\mathbf{1 0 0} \\
\mathbf{\%}\end{array}$ & Total & & \\
\hline
\end{tabular}


Table (2): Demographic characteristics of the examined criminal and civil cases with their statistical significance tests:

\begin{tabular}{|c|c|c|c|c|}
\hline $\begin{array}{l}\text { Groups } \\
\text { Socio-demographic data }\end{array}$ & Criminal (84) & Civil(516) & $\begin{array}{l}\text { Statistical } \\
\text { Significance tests }\end{array}$ & $P$ value \\
\hline Age $($ mean \pm SD) & $33.38 \pm 9.57$ & $\begin{array}{l}36.83 \pm 18.1 \\
2\end{array}$ & $\begin{array}{l}\text { student } \mathrm{t} \text { test }= \\
1.70\end{array}$ & $P$ value $=0.089$ \\
\hline $\begin{array}{l}\text { Sex: } \\
\begin{array}{l}\text { - Male } \\
\text { - Female }\end{array}\end{array}$ & $\begin{array}{l}77(91.7 \%) \\
7(8.3 \%)\end{array}$ & $\begin{array}{l}318(61.6 \% \\
) \\
198(38.4 \% \\
)\end{array}$ & $\chi^{2}=28.98$ & $\begin{array}{l}\mathrm{P} \\
\text { value }=0.000^{*}\end{array}$ \\
\hline $\begin{array}{l}\text { Education: } \\
\text { • lower than } \\
\text { secondary school } \\
\text { • secondary school } \\
\text { and technical } \\
\text { institute } \\
\text { - collage and post } \\
\text { graduate }\end{array}$ & $\begin{array}{l}31(36.9 \%) \\
37(44.0 \%) \\
16(19.1 \%)\end{array}$ & $\begin{array}{l}235 \\
(45.5 \%) \\
167 \\
(32.4 \%) \\
114 \\
(22.1 \%)\end{array}$ & $\chi^{2}=4.43$ & $P$ value $=0.109$ \\
\hline $\begin{array}{l}\text { Occupation: } \\
\qquad \begin{array}{l}\text { • employed } \\
\text { • unemployed }\end{array}\end{array}$ & $\begin{array}{l}27(32.1 \%) \\
57(67.9 \%)\end{array}$ & $\begin{array}{l}53(10.3 \%) \\
463 \\
(89.7 \%)\end{array}$ & $\chi^{2}=29.9$ & $\begin{array}{l}\mathrm{P} \\
\text { value }=0.000 *\end{array}$ \\
\hline $\begin{array}{c}\text { Marital status: } \\
\text { - single } \\
\text { - widow } \\
\text { - divorced } \\
\text { - married }\end{array}$ & $\begin{array}{l}42(50.0 \%) \\
0(0.0 \%) \\
15(17.9 \%) \\
27(32.1 \%)\end{array}$ & $\begin{array}{l}344(66.7 \% \\
) \\
22(4.2 \%) \\
50(9.7 \%) \\
100(19.4 \% \\
)\end{array}$ & $\begin{array}{l}\text { Fisher 's exact= } \\
23.6\end{array}$ & $\begin{array}{l}\mathrm{P} \\
\text { value }=0.000 *\end{array}$ \\
\hline
\end{tabular}

*significant difference ( $\mathrm{p}$ value $<0.05)$. 
Table (3): Difference between the examined criminal and civil cases regarding the diagnostic parameters with their statistical significance Chisquare test:

\begin{tabular}{|c|c|c|c|c|}
\hline $\begin{array}{l}\text { Groups } \\
\text { Items }\end{array}$ & $\begin{array}{l}\text { Criminal } \\
(84)\end{array}$ & $\begin{array}{l}\text { Civil } \\
\text { (516) }\end{array}$ & $\begin{array}{l}\text { Chi- } \\
\text { squar } \\
\text { e test }\end{array}$ & $\begin{array}{l}P \text { - } \\
\text { value }\end{array}$ \\
\hline $\begin{array}{l}\text { diagnosis: } \\
\text { - Intellectual disability } \\
\text { - Schizophrenia } \\
\text { - Delusional disorder } \\
\text { - Schizoaffective disorder } \\
\text { - Bipolar I disorder } \\
\text { - Major depressive disorder } \\
\text { - Insomnia disorder } \\
\text { - Neurocognitive disorder } \\
\text { - Personality disorder } \\
\text { - Coma } \\
\text { - Comorbidity with substance use disorder } \\
\text { - Comorbidity with personality disorder } \\
\text { - Intellectual disability with Bipolar I } \\
\text { disorder } \\
\text { - Other comorbidities (dual diagnosis) }\end{array}$ & $\begin{array}{l}3(3.6 \%) \\
21(25.0 \%) \\
5(6.0 \%) \\
3(3.6 \%) \\
12(14.3 \%) \\
1(1.2 \%) \\
0(0.0 \%) \\
0(0.0 \%) \\
3(3.6 \%) \\
0(0.0 \%) \\
28(33.3 \%) \\
7(8.3 \%) \\
1(1.2 \%) \\
0(0.0 \%)\end{array}$ & $\begin{array}{l}292(56.6 \\
\%) \\
55(10.7 \%) \\
1(0.2 \%) \\
14(2.7 \%) \\
12(2.3 \%) \\
12(2.3 \%) \\
1(0.2 \%) \\
84(16.3 \%) \\
3(0.6 \%) \\
8(1.6 \%) \\
1(0.2 \%) \\
1(0.2 \%) \\
0(0.0 \%) \\
32(6.2 \%)\end{array}$ & $\begin{array}{l}\chi^{2}= \\
336.0\end{array}$ & $\begin{array}{l}\mathrm{P} \\
\text { value }= \\
0.000^{*}\end{array}$ \\
\hline
\end{tabular}

*significant difference ( $\mathrm{p}$ value $<0.05)$. 
Table (4): Substance abuse of the examined criminal and civil cases with its statistical significance Chi-square test:

\begin{tabular}{|c|l|l|l|l|}
\hline $\begin{array}{l}\text { Groups } \\
\text { Substance abuse }\end{array}$ & $\begin{array}{l}\text { Criminal } \\
(\mathbf{8 4})\end{array}$ & Civil(516) & $\begin{array}{l}\text { Chi- } \\
\text { square } \\
\text { test }\end{array}$ & P - value \\
\hline $\begin{array}{c}\text { Substance abuse: } \\
\text { - no } \\
\text { - yes }\end{array}$ & $\begin{array}{l}46(54.8 \%) \\
38(45.2 \%)\end{array}$ & $\begin{array}{l}507(98.3 \%) \\
9(1.7 \%)\end{array}$ & $\chi^{2}=189.2$ & $\begin{array}{l}\mathrm{P} \\
\text { value }=0.000^{*}\end{array}$ \\
\hline
\end{tabular}

*significant difference ( $\mathrm{p}$ value $<0.05)$.

Table (5): Past history of mental illness of the examined criminal and civil cases with their statistical significance tests (Student $t$ test and Chi-square test)

\begin{tabular}{|c|c|c|c|c|}
\hline Groups Item & $\begin{array}{c}\text { Criminal } \\
(84)\end{array}$ & $\begin{array}{l}\text { Civil } \\
(516)\end{array}$ & $\begin{array}{c}\text { statistical } \\
\text { significance tests } \\
\text { (Student t test } \\
\text { and Chi-square } \\
\text { test) }\end{array}$ & $P$ - value \\
\hline $\begin{array}{l}\text { Duration of } \\
\text { illness } \\
(\text { mean } \pm S D)\end{array}$ & $12.67 \pm 9.03$ & $21.87 \pm 11.92$ & $\begin{array}{l}\text { student } t \text { test }= \\
6.71\end{array}$ & $\begin{array}{l}\mathrm{P} \\
\text { value }=0.000 \\
*\end{array}$ \\
\hline $\begin{array}{r}\text { Past admission: } \\
\qquad \text { yes } \\
\text { - no }\end{array}$ & $\begin{array}{l}50(59.5 \%) \\
34(40.5 \%)\end{array}$ & $\begin{array}{l}124(24.0 \%) \\
392(76.0 \%)\end{array}$ & $\chi^{2}=44.19$ & $\begin{array}{l}\mathrm{P} \\
\text { value }=0.000 \\
*\end{array}$ \\
\hline $\begin{array}{c}\text { Compliance on } \\
\text { treatment: } \\
\text { - yes } \\
\text { - no }\end{array}$ & $\begin{array}{l}7(13.5 \%) \\
45(86.5 \%)\end{array}$ & $\begin{array}{l}138(65.1 \%) \\
74(34.9 \%)\end{array}$ & $\chi^{2}=44.96$ & $\begin{array}{l}\mathrm{P} \\
\text { value }=0.000 \\
*\end{array}$ \\
\hline
\end{tabular}

*significant difference ( $\mathrm{p}$ value $<0.05)$. 
Table (6): Past criminal history of the examined criminal and civil cases with its statistical significance Chi-square test:

\begin{tabular}{|c|c|c|c|l|}
\hline $\begin{array}{l}\text { Groups } \\
\text { Past forensic history: }\end{array}$ & $\begin{array}{c}\text { Criminal } \\
\mathbf{( 8 4 )}\end{array}$ & $\begin{array}{c}\text { Civil } \\
\mathbf{( 5 1 6 )}\end{array}$ & $\begin{array}{c}\text { Chi-square } \\
\text { test }\end{array}$ & \multicolumn{1}{|l}{ - value } \\
\hline $\begin{array}{c}\text { Past criminal history: } \\
\text { - no }\end{array}$ & $\begin{array}{l}39(46.4 \%) \\
\text { - yes }\end{array}$ & $\begin{array}{l}481(93.2 \%) \\
35(53.6 \%)\end{array}$ & $\begin{array}{l}\chi^{2}=136.86 \\
\mathrm{P}\end{array}$ & $\mathrm{P}$ \\
value $=0.000^{*}$ \\
\hline
\end{tabular}

*significant difference ( $\mathrm{p}$ value $<0.05$ ).

Table (7): Family history of the examined criminal and civil cases with its statistical significance Chi- square test:

\begin{tabular}{|c|c|c|c|c|}
\hline $\begin{array}{l}\text { Family history } \\
\text { Items }\end{array}$ & $\begin{array}{l}\text { Criminal } \\
(84)\end{array}$ & Civil (516) & $\begin{array}{l}\text { Chi- } \\
\text { square test }\end{array}$ & $P$ - value \\
\hline $\begin{array}{l}\text { Family history of mental } \\
\text { illness: } \\
\qquad \begin{aligned} & \\
& \bullet \text { yes } \\
& \text { no }\end{aligned}\end{array}$ & $\begin{array}{l}45(53.6 \%) \\
39(46.4 \%)\end{array}$ & $\begin{array}{l}179(34.7 \%) \\
337(65.3 \%)\end{array}$ & $\chi^{2}=11.00$ & $\begin{array}{l}\mathrm{P} \\
\text { value }=0.00 \\
1^{*}\end{array}$ \\
\hline $\begin{array}{r}\text { Family history of } \\
\text { substance abuse: } \\
\bullet \quad \text { yes } \\
\bullet \text { no }\end{array}$ & $\begin{array}{l}12(14.3 \%) \\
72(85.7 \%)\end{array}$ & $\begin{array}{l}2(0.4 \%) \\
514(99.6 \%)\end{array}$ & $\chi^{2}=61.23$ & $\begin{array}{l}\mathrm{P} \\
\text { value }=0.00 \\
0^{*}\end{array}$ \\
\hline $\begin{array}{r}\text { Family history of crimes: } \\
\qquad \begin{aligned} \bullet & \text { yes } \\
\bullet & \text { no }\end{aligned}\end{array}$ & $\begin{array}{l}8(9.5 \%) \\
76(90.5 \%)\end{array}$ & $\begin{array}{l}1(0.2 \%) \\
515(99.8 \%)\end{array}$ & $\chi^{2}=42.56$ & $\begin{array}{l}\mathrm{P} \\
\text { value }=0.00 \\
0 *\end{array}$ \\
\hline $\begin{aligned} \text { Social support: } \\
$\[ \text { good } \]$ \\
\bullet \text { moderate } \\
\bullet \quad \text { no support }\end{aligned}$ & $\begin{array}{l}33(39.3 \%) \\
15(17.9 \%) \\
36(42.9 \%)\end{array}$ & $\begin{array}{l}446(86.2 \%) \\
37(7.2 \%) \\
34(6.6 \%)\end{array}$ & $\chi^{2}=111.0$ & $\begin{array}{l}\mathrm{P} \\
\text { value }=0.00 \\
0 *\end{array}$ \\
\hline
\end{tabular}


Table (8): Opinion of the forensic psychiatry committee about the criminal responsibility of the examined criminal cases

\begin{tabular}{|l|c|c|c|}
\hline \multirow{2}{*}{ Type of crime } & \multicolumn{2}{|l|}{} \\
\cline { 2 - 3 } & \multirow{2}{*}{ Opinion of committee } & \multirow{2}{*}{ Total } \\
\cline { 2 - 3 } & & $\begin{array}{c}\text { Non- } \\
\text { responsible }\end{array}$ & \\
\hline Homicide (murder, & & & \\
\hline Aggranticide) & $4(80.0 \%)$ & $1(20.0 \%)$ & $\mathbf{5 ( 1 0 0 \% )}$ \\
\hline Rape and sexual assault & $6(100 \%)$ & $0(0.0 \%)$ & $\mathbf{6 ( 1 0 0 \% )}$ \\
\hline Kidnapping & $2(100 \%)$ & $0(0.0 \%)$ & $\mathbf{2 ( 1 0 0 \% )}$ \\
\hline Threat (menace) & $1(25.0 \%)$ & $3(75.0 \%)$ & $\mathbf{4 ( 1 0 0 \% )}$ \\
\hline Attempt of suicide & $2(50.0 \%)$ & $2(50.0 \%)$ & $\mathbf{4 ( 1 0 0 \% )}$ \\
\hline Offences against properties, & $6(35.3 \%)$ & $11(64.7 \%)$ & $\mathbf{1 7 ( 1 0 0 \% )}$ \\
\hline Robbery & $2(50.0 \%)$ & $2(50.0 \%)$ & $\mathbf{4 ( 1 0 0 \% )}$ \\
\hline Financial crime & $3(75.0 \%)$ & $1(25.0 \%)$ & $\mathbf{4 ( 1 0 0 \% )}$ \\
\hline Simple assault & $8(47.1 \%)$ & $9(52.9 \%)$ & $\mathbf{1 7}(\mathbf{1 0 0 \% )}$ \\
\hline Political crimes & $3(42.9 \%)$ & $4(57.1 \%)$ & $\mathbf{7 ( 1 0 0 \% )}$ \\
\hline Other crimes & $3(42.9 \%)$ & $4(57.1 \%)$ & $\mathbf{7 ( 1 0 0 \% )}$ \\
\hline Total & $\mathbf{4 1 ( 4 8 . 8 )}$ & $\mathbf{4 3 ( 5 1 . 2 \% )}$ & $\mathbf{8 4 ( 1 0 0 \% )}$ \\
\hline
\end{tabular}


Table (9): The relation between criminal responsibility and diagnosis of the offenders in the examined criminal cases with their statistical significance Fisher exact test:

\begin{tabular}{|c|c|c|c|}
\hline \multirow[b]{2}{*}{ Diagnosis } & \multicolumn{2}{|c|}{ Opinion of committee } & \multirow[b]{2}{*}{ Total } \\
\hline & Responsible & $\begin{array}{c}\text { Non- } \\
\text { responsible }\end{array}$ & \\
\hline Schizophrenia & $\begin{array}{c}4 \\
19 \%\end{array}$ & $\begin{array}{c}17 \\
81 \%\end{array}$ & $\begin{array}{c}21 \\
100 \%\end{array}$ \\
\hline Bipolar I disorder & $\begin{array}{c}2 \\
16.7 \%\end{array}$ & $\begin{array}{c}10 \\
83.3 \%\end{array}$ & $\begin{array}{c}12 \\
100 \%\end{array}$ \\
\hline Delusional disorder & $\begin{array}{c}0 \\
0.0 \%\end{array}$ & $\begin{array}{c}5 \\
100 \%\end{array}$ & $\begin{array}{c}5 \\
100 \%\end{array}$ \\
\hline Schizoaffective disorder & $\begin{array}{c}2 \\
66.7 \%\end{array}$ & $\begin{array}{c}1 \\
33.3 \%\end{array}$ & $\begin{array}{c}3 \\
100 \%\end{array}$ \\
\hline Intellectual disability & $\begin{array}{c}2 \\
66.7 \%\end{array}$ & $\begin{array}{c}1 \\
33.3 \%\end{array}$ & $\begin{array}{c}3 \\
100 \%\end{array}$ \\
\hline Major depressive disorder & $\begin{array}{c}1 \\
100 \%\end{array}$ & $\begin{array}{c}0 \\
0.0 \%\end{array}$ & $\begin{array}{c}1 \\
100 \%\end{array}$ \\
\hline Personality disorders & $\begin{array}{c}3 \\
100 \%\end{array}$ & $\begin{array}{c}0 \\
0.0 \%\end{array}$ & $\begin{array}{c}3 \\
100 \%\end{array}$ \\
\hline $\begin{array}{c}\text { Comorbidity with } \\
\text { Substance use disorder }\end{array}$ & $\begin{array}{c}22 \\
78.6 \%\end{array}$ & $\begin{array}{c}6 \\
21.4 \%\end{array}$ & $\begin{array}{c}28 \\
100 \%\end{array}$ \\
\hline $\begin{array}{l}\text { Comorbidity with } \\
\text { Personality disorder }\end{array}$ & $\begin{array}{c}5 \\
71.4 \%\end{array}$ & $\begin{array}{c}2 \\
28.6 \%\end{array}$ & $\begin{array}{c}7 \\
100 \%\end{array}$ \\
\hline $\begin{array}{c}\text { Intellectual disability with } \\
\text { Bipolar I disorder }\end{array}$ & $\begin{array}{c}0 \\
0.0 \%\end{array}$ & $\begin{array}{c}1 \\
100 \%\end{array}$ & $\begin{array}{c}1 \\
100 \%\end{array}$ \\
\hline Total & $\begin{array}{c}41 \\
48.8 \%\end{array}$ & $\begin{array}{c}43 \\
51.2 \%\end{array}$ & $\begin{array}{c}84 \\
100 \%\end{array}$ \\
\hline $\begin{array}{c}\text { Fisher exact test } \\
\text { P value }\end{array}$ & & $\begin{array}{c}34.24 \\
0.000 *\end{array}$ & \\
\hline
\end{tabular}

*Significant difference ( $\mathrm{p}$ value $<0.05$ ). 
Table (10): The relation between criminal responsibility and the state of mental illness in the examined criminal cases with their statistical significance Chi- square test:

\begin{tabular}{|c|c|c|c|c|}
\hline $\begin{array}{l}\text { Opinion of committee } \\
\text { Items }\end{array}$ & $\begin{array}{l}\text { Responsible } \\
\text { (41) }\end{array}$ & $\begin{array}{l}\text { Non-responsible } \\
\text { (43) }\end{array}$ & $\begin{array}{l}\text { Chi- } \\
\text { square test }\end{array}$ & P- value \\
\hline $\begin{array}{l}\text { Severity of illness: } \\
\text {-Mild } \\
\text {-Moderate } \\
\text {-Sever } \\
\text { - Severe with psychotic } \\
\text { features }\end{array}$ & $\begin{array}{l}14(34.1 \%) \\
12(29.3 \%) \\
12(29.3 \%) \\
3(7.3 \%)\end{array}$ & $\begin{array}{l}1(2.3 \%) \\
17(39.5 \%) \\
18(41.9 \%) \\
7(16.3 \%)\end{array}$ & $\chi^{2}=14.89$ & $\begin{array}{l}\mathrm{P} \\
\text { value }=0 . \\
002 *\end{array}$ \\
\hline $\begin{array}{l}\text { Degree of cognitive } \\
\text { impairment } \\
\text {-Mild } \\
\text {-Moderate } \\
\text {-Sever } \\
\text {-No impairment }\end{array}$ & $\begin{array}{l}21(51.2 \%) \\
3(7.3 \%) \\
0(0.0 \%) \\
17(41.5 \%)\end{array}$ & $\begin{array}{l}25(58.1 \%) \\
8(18.6 \%) \\
2(4.7 \%) \\
8(18.6 \%)\end{array}$ & $\chi^{2}=7.82$ & $\begin{array}{l}\mathrm{P} \\
\text { value }=0 . \\
050 *\end{array}$ \\
\hline $\begin{array}{l}\text { impairment of testing } \\
\text { reality: } \\
\text { Yes } \\
\text { No }\end{array}$ & $\begin{array}{l}5(12.2 \%) \\
36(87.8 \%)\end{array}$ & $\begin{array}{l}36(83.7 \%) \\
7(16.3 \%)\end{array}$ & $\chi^{2}=42.97$ & $\begin{array}{l}\mathrm{P} \\
\text { value }=0 . \\
000 *\end{array}$ \\
\hline $\begin{array}{l}\text { impairment of self- } \\
\text { control: } \\
\text { Yes } \\
\text { No }\end{array}$ & $\begin{array}{l}7(17.1 \%) \\
34(82.9 \%)\end{array}$ & $\begin{array}{l}25(58.1 \%) \\
18(41.9 \%)\end{array}$ & $\chi^{2}=15.01$ & $\begin{array}{l}\mathrm{P} \\
\text { value }=0 . \\
000 *\end{array}$ \\
\hline $\begin{array}{l}\text { impairment of insight: } \\
\text { Yes } \\
\text { No }\end{array}$ & $\begin{array}{l}19(46.3 \%) \\
22(53.7 \%)\end{array}$ & $\begin{array}{l}42(97.7 \%) \\
1(2.3 \%)\end{array}$ & $\chi^{2}=27.81$ & $\begin{array}{l}\mathrm{P} \\
\text { value }=0 . \\
000 *\end{array}$ \\
\hline
\end{tabular}

*significant difference ( $\mathrm{p}$ value $<0.05)$. 
Table (11): Opinion of the committee in cases of guardianship, child custody and nullity of contract

\begin{tabular}{|c|c|c|c|c|c|}
\hline \multirow[b]{3}{*}{$\begin{array}{l}\text { Type of civil } \\
\text { competency }\end{array}$} & \multicolumn{4}{|c|}{ Opinion of committee } & \multirow[b]{3}{*}{ Total } \\
\hline & \multirow[b]{2}{*}{ Competent } & \multicolumn{2}{|c|}{ Non-competent } & \multirow[b]{2}{*}{\begin{tabular}{|c|} 
to be \\
evaluated \\
again after 6 \\
month
\end{tabular}} & \\
\hline & & $\begin{array}{c}\text { No ability } \\
\text { to be } \\
\text { competent } \\
\text { in the future }\end{array}$ & $\begin{array}{l}\text { May be } \\
\text { competent } \\
\text { later on }\end{array}$ & & \\
\hline \multirow{2}{*}{ Guardianship } & \multirow{2}{*}{$68(14.2 \%)$} & \multicolumn{2}{|c|}{$85.6 \%$} & \multirow{2}{*}{$1(0.2 \%)$} & \multirow{2}{*}{$480(100 \%)$} \\
\hline & & $394(82.1 \%)$ & $17(3.5 \%)$ & & \\
\hline child custody & $3(100 \%)$ & $0(0.0 \%)$ & $0(0.0 \%)$ & $0(0.0 \%)$ & $3(100 \%)$ \\
\hline $\begin{array}{l}\text { nullity of } \\
\text { contract }\end{array}$ & $3(100 \%)$ & $0(0.0 \%)$ & $0(0.0 \%)$ & $0(0.0 \%)$ & $3(100 \%)$ \\
\hline
\end{tabular}

Table (12): Opinion of the committee in cases of guardianship and disability degree

\begin{tabular}{|c|c|c|c|c|}
\hline \multirow{3}{*}{$\begin{array}{l}\text { Type of civil } \\
\text { competency }\end{array}$} & \multicolumn{3}{|c|}{ Opinion of committee } & \multirow{3}{*}{ Total } \\
\hline & \multicolumn{2}{|c|}{ Competent } & \multirow{2}{*}{$\begin{array}{c}\text { Non- } \\
\text { competent }\end{array}$} & \\
\hline & $\begin{array}{c}\text { Disability degree } \\
10-20 \%\end{array}$ & $\begin{array}{c}\text { Disability } \\
\text { degree } \\
20-50 \%\end{array}$ & & \\
\hline $\begin{array}{l}\text { Guardianship \& } \\
\text { Disability degree }\end{array}$ & $3(11.5 \%)$ & $3(11.5 \%)$ & $20(77 \%)$ & $26(100 \%)$ \\
\hline
\end{tabular}


Table (13): Opinion of the committee in cases of fitness for work and disability degree

\begin{tabular}{|l|c|c|c|}
\hline \multirow{2}{*}{$\begin{array}{l}\text { Type of civil } \\
\text { competency }\end{array}$} & Fit for work & Not-fit for work & \multirow{2}{*}{ Total } \\
\cline { 2 - 4 } $\begin{array}{l}\text { Fitness for work \& } \\
\text { Disability degree }\end{array}$ & $3(75 \%)$ & $1(25 \%)$ & $4(100 \%)$ \\
\hline
\end{tabular}

* In all of these cases the disability degree was $10-20 \%$.

Table (14): The relation between civil responsibility (competency) and the diagnosis in the examined guardianship cases

\begin{tabular}{|c|c|c|c|c|}
\hline \multirow[b]{2}{*}{ Diagnosis } & \multicolumn{3}{|c|}{$\begin{array}{l}\text { Opinion of the committee in } \\
\text { guardianship cases }\end{array}$} & \multirow[b]{2}{*}{ Total } \\
\hline & competent & $\begin{array}{c}\text { non- } \\
\text { competent }\end{array}$ & $\begin{array}{r}\text { To be } \\
\text { evaluated } \\
\text { after } 6 \\
\text { months }\end{array}$ & \\
\hline Intellectual disability & 22 & 253 & 0 & 275 \\
\hline Schizophrenia & 18 & 35 & 0 & 53 \\
\hline Schizoaffective disorder & 6 & 7 & 0 & 13 \\
\hline Bipolar I disorder & 5 & 3 & 0 & 8 \\
\hline Major depressive disorder & 8 & 0 & 1 & 9 \\
\hline Insomnia disorder & 1 & 0 & 0 & 1 \\
\hline Neurocognitive disorder & 6 & 74 & 0 & 80 \\
\hline Personality disorder & 1 & 0 & 0 & 1 \\
\hline Coma & 0 & 8 & 0 & 8 \\
\hline $\begin{array}{r}\text { Comorbidity with } \\
\text { substance use disorder }\end{array}$ & 0 & 1 & 0 & 1 \\
\hline $\begin{array}{r}\text { Other comorbidities (dual } \\
\text { diagnosis) }\end{array}$ & 1 & 30 & 0 & 31 \\
\hline Total & 68 & 411 & 1 & 480 \\
\hline
\end{tabular}


Table (15): The relation between severity of mental illness and degree of cognitive impairment with the civil responsibility (competency) in the examined guardianship cases with its statistical significance Chi- square test:

\begin{tabular}{|r|c|c|c|}
\hline Opinion of committee & Competent & Non competent & Significant \\
Items & $(68)$ & $(411)$ & test \\
\hline Severity of illness: & & & Chi square \\
-Mild & $56(82.3 \%)$ & $47(11.4 \%)$ & test $=$ \\
-Moderate & $5(7.4 \%)$ & $160(38.9 \%)$ & 201.9 \\
- -Sever & $2(2.9 \%)$ & $181(44 \%)$ & $\mathrm{P}$ \\
-with psychotic features & $1(1.5 \%)$ & $4(1 \%)$ & value $=0.000 *$ \\
-in full remission & $4(5.9 \%)$ & $1(0.3 \%)$ & \\
-with behavioral disturbance & $0(0.0 \%)$ & $18(4.4 \%)$ & \\
Degree of cognitive impairment & & & Chi square \\
-Mild & $46(67.6 \%)$ & $52(12.7 \%)$ & test $=$ \\
-Moderate & $4(5.9 \%)$ & $173(42.1 \%)$ & 233.7 \\
-Sever & $0(0.0 \%)$ & $184(44.7 \%)$ & $\mathrm{P}$ \\
-No impairment & $18(26.5 \%)$ & $2(0.5 \%)$ & value $=0.000 *$ \\
\hline
\end{tabular}

NB. One case had severe illness and with no cognitive impairment to be evaluated again after 6 month

*significant difference ( $\mathrm{p}$ value $<0.05$ ).

\section{DISCUSSION}

A forensic psychiatrist is usually asked to determine the responsibility of the person who committed a crime, or his fitness for trial in case of criminal proceedings. In case of civil proceedings, he evaluates the fitness of the person for performing all civil affairs (Chadda et al., 2002).

In this study most of cases $(86 \%)$ presented with civil problems, while $14 \%$ of the cases presented with criminal problems.

These results were in agreement with those of Mohammed et al. (2012) where they studied the criminal and civil problems associated with mental disorders in Assiut Governorate during the period from 2005-2010.
They found that, the majority of their studied cases (159 cases) were presented with civil problems and only 4 cases were presented with criminal problems.

Concerning the demographic characteristics of the examined criminal and civil cases in the current work, most of criminal and civil cases were males (65.8\%). Their mean age was $33.38 \pm 9.57$ years in criminal cases and $36.83 \pm$ 18.12 years in civil cases. As regarding the educational level, most of the cases had secondary $(34 \%)$ or less than secondary school educational level (44.3\%). Moreover, most of criminal and civil cases were unemployed $(86.7 \%)$ and single (64.3\%). 
These results were in agreement with those of Elsayed et al. (2010) where they studied the characteristics of mentally ill offenders in Saudi Arabia they found that, $93 \%$ of cases were males and only $7 \%$ were females, $73 \%$ of cases were from 20 to 40 years old and, a significant percentage of mentally ill offenders were single (64\%) and unemployed (34\%).

Similarly, Hartwell (2004), in his study on 701 mentally ill offenders, found that $81 \%$ of the cases were males, while $19 \%$ were females and $63 \%$ of the cases were from 27 to 45 years old. $63 \%$ of the cases were in a level less than high school, $27 \%$ were in high school or general education diploma and $10 \%$ were in a level more than high school.

The obtained results of the current study where most of criminal cases were males could be explained according to the WHO study by the higher rate for alcohol-related disorders and other substances use disorder among males (Sadock et al., 2015).

Furthermore, Fisher and Drake (2007) stated that substance abuse is an indirect pathway from symptoms of mental disorders to crime.

Regarding to the relation between unemployment and unmarried states with crimes, many studies found that there was a high rate of unemployment and unmarried states in mentally ill offenders as well as in offenders without psychiatric disorders, which may be attributed to increased unemployment rates and delayed marital age in the Middle East region (Menezes et al., 2007).

In addition, Lurigio (2012) stated that, because of their illnesses, mentally disordered people have more difficulties to finish education or maintain a job, which in turn complicates upward social mobility. Living in poverty and with no legitimate opportunities for advancement, people with mental disorders have no other choice than to engage in criminal activity.

The demographic characteristics of the civil cases in the current study were in agreement with the results of Mohammed et al. (2012) when they studied civil problems associated with mental disorders in Assiut Governorate, they found that the highest frequency of their cases was in the middle age group from $20 \leq 30$ years, with male predominance. Males represent about triple the number of females, most cases presented with civil problems were not educated, unemployed and single.

Regarding the diagnosis of the examined criminal and civil cases, most criminal cases $(33.3 \%)$ had comorbidity with substance abuse, followed by schizophrenia $(25 \%)$ and bipolar I disorder (14.3\%) while, most of civil cases (56.6) had intellectual disability, followed by neurocognitive 
disorder (16.3\%) and lastly schizophrenia (10.7\%).

This result was in agreement with results of Elsayed et al. (2010) where they found that, dual diagnosis represented $39 \%$ of the total mentally disordered offenders of their studied cases. Hartwell, (2004) found in his study a higher rate $(62 \%)$ of mentally disordered criminal offenders had dual diagnoses. Furthermore, the diagnosis of civil cases was in agreement with those of Mohammed et al. (2012) as they found in their study that, the most common diagnosis in civil cases was intellectual disability, followed by schizophrenia, post traumatic psychosis and neurocognitive disorder.

On the same base, Scott et al. (1998) found that, patients with a dual diagnosis were six times more likely to describe hostile behavior than those with psychosis alone. Wright et al. (2000) declared that mentally-disordered persons with dual diagnosis were considered a high-risk group for committing crimes.

There is evidence that compliance with treatment is particularly poor in those with dual diagnosis and that, the combination of substance misuse and non-compliance with medication in the mentally ill is associated with a high risk of violence (Owen et al., 1996 and Swartz et al., 1998).

Elbogen and Johnson (2009) pointed to the increased risk for violence among persons having mental disorder comorbid with substance use disorder is primarily attributable to substance use disorders and antisocial personality features and not to mental illnesses.

Regarding substance abuse and crimes, the relationship between substance abuse and crime has been proved through many studies, Fisher and Drake (2007) stated that substance abuse is considered as an indirect pathway from symptoms of mental disorders to crime.

According to the current study, the rate of substance abuse among criminal cases was $45.2 \%$.

Lurigio (2012) explained such findings by, the attempts of mentally-disordered person to selfmedicate themselves with drugs or alcohol to reduce the impact of their untreated psychiatric symptoms or to decrease the debilitating side effects of antipsychotic medications that may be the reason of involvement of those persons in criminal behavior.

Concerning the past history of mental illness of the examined criminal and civil cases and in spite of long duration of illness in most cases, there was a high rate of non-admission in psychiatric hospital before $(71 \%)$ and a high percentage of non-compliant on treatment among the cases $(19.8 \%)$. These results indicate that, avoidance or non- awareness of the families about the importance of noticing any 
apparent psychiatric symptoms and seeking medical and psychiatric care for their members, this problem may be attributed to the stigma of psychiatric illness among our countries.

These results in agreement with results of Elsayed et al. (2010) who found that, $58 \%$ of cases of mentally disordered offenders had previous contact with psychiatric services while, according to the study of Hartwell (2004) previous use of clinical services was $71 \%$ of mentally ill offenders.

In the present study, most of cases $(56 \%)$ had no regular contact with mental health service and didn't receive any treatment for their mental illness this result may be explained by the high percentage of intellectual disability (49.2\%) among the studied cases, those patients need rehabilitation programs. Sechoaro et al. (2014) studied the rehabilitation effects in persons having intellectual disability and they found that, the rehabilitation had positive effects on persons having intellectual disability in daily living activities, self-care skills, communication skills and cognitive achievement.

Regarding past criminal history of the examined criminal and civil cases, The high rate of past history of crimes in criminal cases in the current study was in agreement with Fulwiler et al. (1997) study, in which $68 \%$ of chronic mentally ill offenders had past history of similar crimes.
Kupfer et al., (2008) stated that the risk for future violence and criminality is increased in cases of young males with lower socioeconomic and educational level especially when associated with criminal history, juvenile delinquency, traits of antisocial and narcissistic personality disorders, physical abuse or having a criminal parent who was a substance abuser. Current psychiatric symptoms, anger, substance abuse and violent fantasies with a specific target are predictive of offending.

On studying the family history of the examined criminal and civil cases, most criminal cases (53.6\%) had family history of mental illness while, most civil cases (65.3\%) had no family history of mental illness. Family history of substance abuse and family history of crimes were presented in criminal cases in high percentages than in civil cases. High percentage of criminal cases $(42.9 \%)$ had no social support while, most of civil cases $(86.2 \%)$ had good social support.

Sadock et al. (2015) stated that, a genetic, heritable component of mental disorders has been consistently supported through the study of families with the use of population genetic methods over the last 50 years. Moreover, specific chromosomal regions and genes are associated with particular diagnoses have been revealed by more recent techniques in molecular biology. 
Similarly, Kupfer et al. (2008) reported that the risk of violence and criminality is increased in cases having a criminal parent who was a substance abuser.

Furthermore, Nielson et al. (1994) found preliminary evidence that patients with impulsive Aggressive behavior were found to have disturbance in coding for tryptophan hydroxylase and the rate-limiting enzyme in serotonin synthesis. More recently, significantly higher levels of hostility in schizophrenic patients has been associated with a polymorphism in the catechol Omethyltransferase gene on chromosome 22q.

The risk for development of conduct disorder, aggression, and antisocial behavior in children has been found to be increased by having a family history of antisocial personality disorder. Eronen and colleagues (1996) further noted that a family history positive for homicidal ideation and attempts was associated with extreme aggressive acts.

The high percentage of no social support for criminal cases in the present study can be explained by what was previously said by Peterson and Heinz (2016) who stated that, relationships with friends and family can be strained by the untreated mental disorder, as friends and family may not understand or detect untreated symptoms, in this way, mental disorders can lead to social rejection which lead to criminal behavior.

The final opinion of the forensic psychiatry committee in criminal cases in the present study was; 41 cases $(48.8 \%)$ were considered responsible for their crimes, while 43 cases $(51.2 \%)$ were considered non-responsible.

These findings were comparable to those obtained by the study of Elsayed et al. (2010) where, $46 \%$ of the cases were considered fully responsible, $11 \%$ were considered partially responsible, while $33 \%$ were considered non-responsible. They explained the high rate of fully responsible decision by the high rate of substance abuse diagnosis as offenders who were diagnosed to have only substance use disorder were considered full responsible if the person intended to take the substance and knew its prohibited nature.

In the current study, there was a statistically significant difference in the criminal responsibility as regarding diagnosis of the offenders, most cases of schizophrenia, bipolar I disorder, delusional disorder and the case that had intellectual disability comorbid with bipolar I disorder were considered non-responsible, while most cases of schizoaffective disorder, intellectual disability, major depressive disorder, personality disorders, comorbidity with substance use disorder and comorbidity with personality 
disorder were considered responsible.

These results in agreement with Ladikos (1996) who stated that, persons with schizophrenia usually satisfy the criteria of the legal definition of insanity as schizophrenia spectrum and other psychotic disorders were proved to be pathological and endogenous so these disorders are capable of depriving the sufferer of insight and self-control. On the other hand, persons with SubstanceRelated Disorders are not legally considered to be insane as these disorders are neither pathological nor endogenous, nor permanent. Also, personality disorders are not regarded as pathological diseases of the mind and thus as a general rule are not considered to qualify as a mental illness for purposes of the insanity defense.

In the present study, there was also a statistically significant difference in the criminal responsibility according to the severity of mental illness, degree of cognitive impairment, impairment of testing reality, impairment of self-control and impairment of insight.

These results were in agreement with those of Cai et al. (2014) who established "the rating scale of criminal responsibility for mentally disordered offenders (RSCRs)',. The scale consisted of eighteen items. Impairment of insight, impairment of reality testing, and impairment of selfcontrol were included in this scale.
This scale can be applicable for all cases forensic psychiatry.

The opinion of the committee in civil cases in the current study pointed to that, most cases of intellectual disability, schizophrenia, schizoaffective disorder, neurocognitive disorders, coma and cases with dual diagnosis were considered noncompetent while, most cases with major depressive disorder, personality disorders, insomnia disorder were considered competent.

Marson et al. (2006) stated that the cognitive abilities and decisional capacities of persons suffering from serious mental illnesses such as schizophrenia are often significantly be impaired by the reason of their mental illness.

In the current study, there was a statistically significant difference in the civil responsibility (competency) in cases of guardianship according to the severity of mental illness and degree of cognitive impairment.

Campbell (2004) stated that cognition means information processing. It denotes a relatively high level of processing of specific information including thinking, memory, perception, motivation, skilled movements and language.

As regarding the opinion of the committee in cases of fitness for work and disability degree, The cases examined for both fitness for work and disability degree in the current study were four cases, 3 of 
them were considered fit for work as they had mild degree of mental illness, two of them had no cognitive impairment and one case had mild cognitive impairment, while one case was considered not fit for work (had psychotic features and mild degree of cognitive impairment) and the disability degrees were $10-20 \%$ in all of them.

Mental fitness to work is defined as the ability of workers to carry out their work without risks for themselves or others. Mental fitness to work is as important as physical fitness. In many cases, mental disorder may be a cause of disablement (Hessel and Zeiss, 1988; Goetzel et al., 2003).

Elsayed et al. (2009) studied the factors that may have influence on mental fitness for work in persons suffered from mental disorders and found that, the fit group was the younger, the duration of their illness was shorter, the mean hospitalization time was shorter and, the frequency of hospitalization in the last year was less than the unfit group. They explained their findings by the fact that, subjects of this group were considered to be fit for work as the mental illness was less severe in this group of patient which made them had less impairment of performance of their work. However in the unfit group, the duration of illness was longer, the frequency of hospitalization in the last year was more than the fit group, more hospitalization days, there was a disturbed relationship with their colleagues, less productivity, more comorbidity and more diagnosis of schizophrenia. All these factors indicated that the severity of their mental illness was high which affected their performance in work.

Regarding the opinion of the committee in cases of child custody about their diagnosis; one of them had intellectual disability, one had bipolar I disorder, and one case had major depressive disorder. The severity of the disease was mild and there was no cognitive impairment in the three cases, so all of them were considered competent for continued custody of the children. These results were in agreement with Dane and Rosen (2016) who pointed to the fact that, only having a mental disorder in one parent who has the right of custody of

his children is not sufficient to deprive him or her from the child custody. So, the mental illness should be evaluated to determine if it affects parent's behavior by the way that has a negative impact on his or her children. It is the role of family court to search for all evidence and determine whether, the mental disorder of the parent affects badly on the children. Sometimes, mental disorder of the parent may interfere with the ability to care for the child and provide a safe home environment. 
However, not all children will be negatively affected, or affected in the same way, also the influence of mental disorder on child custody competency varies according to the child's age at onset, severity and duration of the mental disorder of the parent.

Regarding the opinion of the committee in cases of nullity of a contract, all cases of nullity of a contract were considered competent, one case had mild intellectual disability and two cases had personality disorders. The opinion of the committee in

\section{CONCLUSIONS}

The psychiatric patients whose problems included involvement with law, their problems were more civil than criminal. The most frequent issue included persons with mental disorders was guardianship. Most of these persons were males, unemployed and single.

Dual diagnosis especially comorbidity with substance use disorder, past history of crimes, family history of mental illness, family history of substance abuse, family history of crimes and absence of social support are risk factors of criminal behavior in mentally disordered persons.

The criminal and civil responsibilities in mentallydisordered persons were affected by the diagnosis, severity of mental illness and the degree of cognitive impairment. the three cases was that in spite of the presence of mental disorders, their awareness at the time of signing the contract in question hadn't been eliminated or reduced and they were aware about the nature and the type of their acts, so they were competent at the time of signing the contract.

The results of the current study in agreement with Slovenko (2004) who stated that, individuals are considered competent to contract if they understand the nature of the contract and its consequences.

\section{RECOMMENDATIONS:}

- Cooperation should be established among the criminal justice, social services, and medical systems to reduce recidivism and victimization among persons with mental disorders.

- prevention of crimes committed by psychiatric patients through:

○ Early detection of psychiatric patients, providing early treatment and management.

○ Rehabilitation programs, occupational therapy and routine regular examination of mentally disordered persons.

- Reducing the incidence of substance abuse is mandatory to reduce the crime rates and should be a focus of attention for service planners. 


\section{REFERENCES}

AAPL, (2005): American Academy of Psychiatry and the Law Ethical Guidelines for the Practice of Forensic Psychiatry. http://www.aapl.org/pdf/ETHI CSGDLNS.pdf.

AAPL, (2015): American Academy of Psychiatry and the Law Practice Guideline for the Forensic Assessment. Journal of the American Academy of Psychiatry and the Law; 43 (2): 3-53

American Psychiatric Association (APA) (2013): Diagnostic and Statistical Manual of Mental Disorders (Fifth ed.). Arlington V. (edt): American Psychiatric Publishing. pp. 525.

Cai W., Zhang Q., Huang F., Guan W., Tang $T$. and Liu C. (2014): The reliability and validity of the rating scale of criminal responsibility for mentally disordered offenders. Forensic Science International; 236: 146-150.

Campbell R. (2004): Campbell's psychiatric dictionary. 8th ed. Oxford: Oxford University Press; p. 131.

Chadda R., Sahu M., Singh R., Gupta A. and Singh T. (2002): Psychiatric assessment on request of external agencies. $\mathbf{J}$ Ment Health Hum Behav; $7: 42-6$.

Dane H. and Rosen J. (2016): View From The Bench:
Parental Mental Health And Child Custody. Family Court Review; 54 (1):10-17.

Elbogen E. and Johnson S. (2009): The intricate link between violence and mental disorder: Results from the National Epidemiologic Survey on Alcohol and Related Conditions. Archives of General Psychiatry; 66: 52161.

Elsayed Y., Al-Zahrani M. and Rashad M. (2009): Factors affecting mental fitness for work in a sample of mentally ill patients. International Journal of Mental Health Systems; 3: 25.

Elsayed Y., AlZahrani M. and Rashad M. (2010): Characteristics of mentally ill offenders from 100 psychiatric court reports. Ann Gen Psychiatry; 9: 4.

Eronen M., Hakola P. and Tiihonen J. (1996): Mental disorders and homicidal behavior in Finland Arch Gen Psychiatry; 53: 497-501.

Fisher W. and Drake R. (2007): Forensic Mental Illness and Other Policy Misadventures. Commentary on "Extending Assertive Community Treatment to Criminal Justice Settings: Origins, Current Evidence, and Future Directions". COMMUNITY MENTAL HEALTH J.; 47: 545- 546.

Fulwiler C., Grossman H., Forbes C. and Ruthazer R. (1997): substance abuse and 
community violence by outpatients with chronic mental illness. Psychiatr Serv.48:1181-1185.

Ghanem M., Gadallah M., Meky F., Mourad S. and El-Kholy G. (2009): National Survey of Prevalence of Mental Disorders in Egypt: preliminary survey. Eastern Mediterranean Health Journal;15 (1): 65-75.

Goetzel R., Hawkins K., Ozminkowski R. and Wang S. (2003): The Health and Productivity Cost Burden of the "Top 10" Physical and Mental Health Conditions Affecting Six Large U.S. Employers in 1999. J Occup Environ Med; 45: 5-14.

Hartwell (2004): Comparison of Offenders with Mental Illness Only and Offenders With Dual Diagnoses. PSYCHIATRIC SERVICES; 55 (2).

Hessel P. and Zeiss E. (1988):

Evaluation of the periodic examination in the South African mining industry. $\mathrm{J}$ Occup Med; 30:580-6.

Klassen P., Wright P. (2006):

Forensic Assessment. In: Goldbloom D. (editor). Psychiatric Clinical Skills. Philadelphia: Mosby Elsevier; pp. 183-98.

Kupfer D., Horner M., Brent D., Lewis D., Reynolds C., Thase M., Travis M., Semple D., Smyth R., Burns J., Darjee R. and McIntosh A. (2008): Oxford American Handbook of Psychiatry, 1st Edition.
Oxford University Press. Pp. 615.

Ladikos A., (1996): A historical overview of crime, mental disorder and the insanity defence. ACTA CRIMINOLOGICA; 9: 102114.

Lurigio A. (2012): Responding to the needs of people with mental illness in the criminal justice system: an area ripe for research and community partnerships. Journal of Crime and Justice; 35(1): 1-12.

Menezes S., Oyebode F. and Haque M. (2007): Mentally disordered offenders in Zimbabwe and in England and Wales: a sociodemographic study. Med Sci Law ;47:253261.

Mohamed F., Abd-El Moneim W., Mohamed Z. and George S. (2012): Legal and medical aspects of mental disorders. CS Thesis at Faculty of Medicine, Assiut University.

Nielson D., Goldman D. and Virkkunen M. (1994): Suicidality and 5hydroxyindoleacetic acid concentration associated with a tryptophan hydroxylase polymorphism. Arch Gen Psychiatry; 51: 34-38

Okasha A. (2003): Mental health services in the Arab world. Arab Stud Quart; 25:112-116.

Owen R., Fischer E., Booth B. and Cuffel B. (1996): Medication noncompliance and substance abuse among patients with 
schizophrenia. Psychiatric

Services; 47, 853-8.

Peterson J. and Heinz K. (2016): Understanding Offenders with Serious Mental Illness in the Criminal Justice System. Mitchell Hamline Law Review; 42: 2- 3.

Sadock B. and Sadock V.(2005): Kaplan \& Sadock's Synopsis of psychiatry. 4th ed. Lippincott Williams \& Wilkins, a Walters Kluwer Company; New York; Pp. 3536.

Sadock B., Sadock V. and Ruiz P. (2015): Kaplan \& Sadock's Synopsis of psychiatry. 11th ed. Lippincott Williams \& Wilkins, a Walters Kluwer Company; New York; Pp. 291. Scott H., Johnson S. and Menezes P. (1998): Substance misuse and risk of aggression and offending among the severely mentally ill. British Journal of Psychiatry; 172: 345-50.

Sechoaro E., Scrooby B. and Koen D. (2014): The effects of rehabilitation on intellectuallydisabled people - a systematic review' Health SA Gesondheid; 19(1): 693.

Slovenko R. (2004): Civil competency. In: The American Psychiatric Publishing Textbook of Forensic Psychiatry. Simon R., and Gold L. (editor); American Psychiatric Publishing, Inc; Londone. Pp. 205.
Swartz M., Swanson J., Hiday V., Borum R., Wagner $\mathrm{H}$., and Burns B. (1998): Violence and severe mental illness: the effects of substance abuse and nonadherence to medication. American Journal of Psychiatry; 155: 226-31.

World Health Organization (2007): Mental health: the bare facts. Retrieved from http://www.who.int/mental_he alth/en/index.html

Wright S., Gournay K., Glorney E. and Thornicroft G. (2000): Dual diagnosis in the suburbs: prevalence,need, and inpatient service use. J Soc Psychiatry Psychiatr Epidemiol.; 35: 297-304.

Youssef H. and Youssef F. (1996): Evidence for the existence of schizophrenia in medieval Islamic society. Hist Psychiatry; 7:55-62. 